\title{
ON CONTINUOUS TIME MODELS IN THE THEORY OF DAMS
}

\author{
J. F. C. KINGMAN
}

(received 23 July 1963)

The problem of storage in an infinite dam with a continuous release has been studied by a number of authors $([5],[3],[2])$, who have formulated it in probabilistic terms by supposing the input to be a continuous time stochastic process. These authors have encountered difficulties which they have overcome by regarding the continuous time problem as a limit of discrete time analogues. The purpose of this paper is to suggest that these difficulties are the result of an unfortunate specification of the problem, and to show that the adoption of a slightly different (and more realistic) formulation avoids the difficulties and allows a treatment which does not have recourse to discrete time analogues.

The input to the dam (in $t>0$ ) is completely determined by the function $X(t)$, where $X(t)$ is the total input during the time interval $(0, t]$. It is usual to describe the fluctuations in the input by supposing $X(t)$ to be a realisation of a stochastic process, but for most of the analysis of this paper this is an irrelevant complication, and we shall regard $X(t)$ simply as a function of $t$. From its definition, $X(t)$ must be right-continuous and nondecreasing in $t \geqq 0$, and must satisfy $X(0)=0$; we make no further assumptions about its behaviour.

Except when the dam is empty, there is a release which we shall suppose to be at constant rate $\alpha>0$. The content of the dam at time $t$ will be denoted by $Z(t)$, and we shall write $Z(0)=z_{0}$. It will be convenient to define a function $\zeta(t)$ by

$$
\zeta(t)=1 \quad \text { if } \quad Z(t)=0, \quad \zeta(t)=0 \quad \text { if } \quad Z(t)>0 .
$$

Thus the values of $t$ for which $\zeta(t)=1$ are exactly the instants at which the dam is empty.

It is reasonable to suppose that, for any $t>0$, the value of $Z(t)$ should depend only on $z_{0}, \alpha$, and the values of $X(s)$ for $s \leqq t$, and the first problem is to determine the form of this dependence. In [2], Gani and Prabhu attempt to do this by remarking in effect that, since during the interval $(t, t+\delta t]$ the dam is non-empty for a time

$$
\tau=\int_{t}^{t+\delta t}\{1-\zeta(s)\} d s
$$


and since therefore the amount released in this interval is equal to $\alpha \tau$, the content $Z(t)$ must satisfy

$Z(t+\delta t)-Z(t)=X(t+\delta t)-X(t)-\alpha \tau=X(t+\delta t)-X(t)-\alpha \int_{t}^{t+\delta t}\{1-\zeta(s)\} d s$.

This equation, which is equation (1.1) of [2], is clearly equivalent to

$$
Z(t)-z_{0}=X(t)-\alpha \int_{0}^{t}\{1-\zeta(s)\} d s,
$$

and so also, if we put

$$
Y(t)=X(t)-\alpha t
$$

to

$$
Z(t)=z_{0}+Y(t)+\alpha \int_{0}^{t} \zeta(s) d s
$$

Because $\zeta$ is defined in terms of $Z$ by (1), this is a non-linear integral equation for $Z$, which one might hope to have exactly one solution. This solution could then very plausibly be taken to represent the content of the dam. Unfortunately, however, there are quite simple inputs for which (3) has no solution at all. Take, for example, $X(t)=\frac{1}{2} \alpha t$, so that (3) becomes

$$
Z(t)=z_{0}-\frac{1}{2} \alpha t+\alpha \int_{0}^{t} \zeta(s) d s,
$$

and suppose that (4) has a (non-negative measurable) solution $Z(t)$. Then $Z$ is differentiable almost everywhere, with

$$
Z^{\prime}(t)=-\frac{1}{2} \alpha+\alpha \zeta(t),
$$

for almost all $t$. Now suppose that this holds for some $t$ for which $\zeta(t)=1$. Then

$$
Z(t)=0, \quad Z^{\prime}(t)=\frac{1}{2} \alpha,
$$

which contradicts the non-negativity of $Z$. Thus $\zeta(t)=0$ for almost all $t$, and substituting back into (4), we get

$$
Z(t)=z_{0}-\frac{1}{2} \alpha t,
$$

which again contradicts $Z \geqq 0$. Hence (4) has no (non-negative measurable) solution.

The reason why (3) breaks down when $X(t)=\frac{1}{2} \alpha t$ can be seen by considering the behaviour of the dam for this case. While the dam is non-empty, there is an input at constant rate $\frac{1}{2} \alpha$ and an output at constant rate $\alpha$, so that the content of the dam decreases steadily at rate $\frac{1}{2} \alpha$. When the dam becomes empty, however, the release ceases, and the content rises at rate $\frac{1}{2} \alpha$. Thus instantaneously the dam becomes non-empty, and the release starts again. We therefore have the picture of a rapid alternation between the states of emptiness and non-emptiness, a picture which is clearly unrealistic and 
which not surprisingly leads to difficulties in the mathematical treatment.

It is surely more natural to suppose that, for this input, the dam content decreases at rate $\frac{1}{2} \alpha$ until it becomes zero, and that thereafter it remains equal to zero, the input rate $\frac{1}{2} \alpha$ being exactly balanced by an equal release rate. For general inputs, it seems reasonable to assume that, when the dam is empty, the release does not entirely cease, but continues at a rate equal to the input rate, so long as this does not exceed $\alpha$.

Thus suppose that there is an input rate $R(t)$, so that

$$
X(t)=\int_{0}^{t} R(s) d s .
$$

Then we assume that the release rate is equal to $\alpha$ if $Z>0$ and to $\min (\alpha, R)$ if $Z=0$. Then it is easy to see that (3) must be replaced by

$$
\begin{aligned}
Z(t) & =z_{0}+Y(t)+\int_{0}^{t} \zeta(s)[\alpha-\min (\alpha, R(s))] d s, \\
& =z_{0}+Y(t)+\int_{0}^{t} \zeta(s)[R(s)-\alpha]-d s,
\end{aligned}
$$

where $x^{+}=\max (x, 0), x^{-}=(-x)^{+}$.

If $A(t)$ is any function of bounded variation in $0 \leqq t<T$ for every $T$, we shall write $A_{+}(t)$ and $A_{-}(t)$ for the total positive and negative variations of $A$ in $(0, t]$. Then, since

$$
Y(t)=\int_{0}^{t}[R(s)-\alpha] d s,
$$

we have

$$
Y_{-}(t)=\int_{0}^{t}[R(s)-\alpha]-d s,
$$

and hence the equation for $Z(t)$ can be written in the form

$$
Z(t)=z_{0}+Y(t)+\int_{0}^{t} \zeta(s) d Y_{-}(s)
$$

We now go on to prove that this modification of the Gani-Prabhu equation (3) has, for any input $X(t)$, a unique solution which can be expressed quite explicitly in terms of $X(t)$, and which can usefully be taken to represent the content of the dam. Thus by using (6) we avoid the difficulties inherent in (3).

The function $Y(t)$ is necessarily right-continuous and of bounded variation, has no downward jumps, and satisfies $Y(0)=0$. More general release rules than those considered here also lead to equations of the form (6) with a function $Y(t)$ having these properties, and we shall therefore formulate our results in such a way as not to assume that $Y(t)$ is expressible in the form (2). The assumption that $Y$ has no downward jumps is, however, essential; if it is not satisfied equation (6) must be modified.

We first prove a lemma about functions of bounded variation. This has 
some connection with some results of Reich [6], but is almost trivial under the very strong conditions which Reich imposes on his functions.

LEMMA 1. Let $A(t)$ be a right-continuous function of bounded variation in $0 \leqq t<T$ which has no upward jumps (i.e. $A(i) \leqq A(t-))$, and suppose that $A(0) \leqq 0$. Write

$$
\mathscr{A}(t)=\left[\sup _{0 \leq s \leq t} A(s)\right]^{+}, \quad E=\{t ; \mathscr{A}(t)=A(t)\} .
$$

Then, for all $t$ in $[0, T]$,

$$
\mathscr{A}(t)=\int_{E \cap(0, t)} d A_{+}(s) .
$$

Proof. We take $A(t)=0$ for $t<0$, so that

$$
\mathscr{A}(t)=\sup _{s \leqq t} A(s) \text {. }
$$

Then, since $A$ has no upward jumps, $\mathscr{A}$ must be continuous. If $t$ belongs to the (countable) discontinuity set $D$ of $A$, then

$$
A(t)<A(t-) \leqq \mathscr{A}(t-)=\mathscr{A}(t),
$$

and so $t \notin E$. Hence $E$ and $D$ are disjoint, and it follows that, if we write

$$
E_{1}=\{t \in[0, T) ; A(t-)=\mathscr{A}(t)\},
$$

then $E_{1}=E_{u} D_{1}$, where $D_{1}$ is a subset of $D$, given by $D_{1}=D_{\cap} E_{1}$.

If $t \notin E_{1}$, then $A(t) \leqq A(t-)<\mathscr{A}(t)$, and hence there exists an open interval $I$ containing $t$ in which $A(s)<\mathscr{A}(t)$. It follows that $\mathscr{A}(s)=\mathscr{A}(t)$ $(s \in I)$, and so $A(s)<\mathscr{A}(s)(s \in I)$, showing that $I$ is disjoint from $E_{1}$. Hence since $0 \in E_{1}$ the complement $E_{1}^{c}$ of $E_{1}$ in $[0, T)$ is open, and every point of $E_{1}^{c}$ has a neighbourhood on which $\mathscr{A}$ is constant. Thus $\mathscr{A}$ is constant on each connected component of $E_{1}^{c}$. But the connected components of $E_{1}^{c}$ form an at most countable family $\left\{I_{n}\right\}$ of open intervals $I_{n}=\left(a_{n}, b_{n}\right)$. Since $\alpha$ is continuous everywhere and constant on $I_{n}$, we have $\mathscr{A}\left(a_{n}\right)=\mathscr{A}\left(b_{n}\right)$, and since $a_{n}, b_{n} \in E_{1}$,

$$
A\left(a_{n}-\right)=\mathscr{A}\left(a_{n}\right)=\mathscr{A}\left(b_{n}\right)=A\left(b_{n}-\right) .
$$

Suppose that $t \in D_{1}=D_{\cap} E_{1}$. Then $A(t)<A(t-)=\mathscr{A}(t)$, and hence, for all sufficiently small $u>0, A(t+u-)<\mathscr{A}(t) \leqq \mathscr{A}(t+u)$, so that $t+u \in E_{1}^{c}$. Thus $t$ is the left end-point of an open interval lying in $E_{1}^{c}$, and hence, since $t \in E_{1}$, we must have $t=a_{n}$ for some $n$. Hence $D_{1} \subseteq\left\{a_{n}\right\} \subseteq E_{1}$, from which it follows that

$$
D_{1}=\left\{a_{n}\right\}_{\cap} D
$$

We can therefore write

$$
[0, T)=E_{\cup} D_{1 \cup} E_{1}^{c}=E_{\cup}\left[\left\{a_{n}\right\}_{n} D\right]_{\cup} \bigcup_{n} I_{n}=E_{\cup} \bigcup_{n} J_{n},
$$


where

$$
\begin{array}{rlll}
J_{n} & =\left(a_{n}, b_{n}\right) & \text { if } & a_{n} \notin D \\
& =\left[a_{n}, b_{n}\right) & \text { if } & a_{n} \in D
\end{array}
$$

Also

$$
\int_{J_{n}} d A(s)=\int_{\left.i a_{n}, b_{n}\right)} d A(s)=A\left(b_{n}-\right)-A\left(a_{n}-\right)=0 .
$$

If $t \in E_{1}$, write $N(t)=\left\{n ; b_{n} \leqq t\right\}$. Then, since $A(0-)=0$,

$\mathscr{A}(t)=A(t-)=\int_{(0, t)} d A(s)=\int_{E_{\Pi}[0, t)} d A(s)+\sum_{N(t)} \int_{J_{n}} d A(s)=\int_{E_{\Pi}(0, t)} d A(s)$.

On the other hand, if $t \notin E_{1}$, then $t \in I_{n}$ for some $n$, and so

$$
\mathscr{A}(t)=\mathscr{A}\left(a_{n}\right)=\int_{E_{\Pi}\left(0, a_{n}\right)} d A(s)=\int_{E_{\cap}[0, t)} d A(s)
$$

since $\left(a_{n}, t\right)$ is disjoint from $E$, and $a_{n} \notin E$ if $a_{n} \in D$. Using the fact that $0 \in E$ only if $A(0)=0$, we see that, for all $t \in[0, T)$,

$$
\mathscr{A}(t)=\int_{E_{\Pi}(0, t)} d A(s)
$$

Since $\mathscr{A}$ is non-decreasing (9) implies that the restriction to $E$ of the Stieltjes measure $\boldsymbol{a}$ determined by $A$ is positive. Hence, if $\mathbf{a}=\boldsymbol{a}^{+}-\boldsymbol{a}^{-}$is the Jordan decomposition of $\boldsymbol{a}$, we must have $\boldsymbol{a}^{-}(E)=0$. Therefore

$$
\mathscr{A}(t)=\int_{E_{\cap}(0, t)} d A(s)=\boldsymbol{a}\left\{E_{\cap}(0, t)\right\}=\boldsymbol{a}^{+}\left\{E_{\cap}(0, t)\right\}=\int_{E_{n}(0, t)} d A_{+}(s),
$$

and the proof is complete.

THEOREM 1. Let $Y(t)$ be a right-continuous function of bounded variation in every finite subinterval of $t \geqq 0$, which has no downward jumps and satisfies $Y(0)=0$, and let $z_{0} \geqq 0$. Then the unique non-negative measurable solution of

$$
Z(t)=z_{0}+Y(t)+\int_{0}^{t} \zeta(s) d Y_{-}(s)
$$

where $\zeta(t)=1$ if $Z(t)=0$ and $\zeta(t)=0$ if $Z(t)>0$, is given by

$$
Z(t)=\max \left[\sup _{0 \leqq \leqslant t}\{Y(t)-Y(s)\}, Y(t)+z_{0}\right]
$$

Proof. If we denote the right hand side of $(10)$ by $Z(t)$, then $Z$ is nonnegative and measurable. The function $A(t)=-Y(t)-z_{0}$ satisfies the conditions of Lemma 1 , and

$$
\begin{aligned}
Z(t) & =z_{0}+Y(t)+\mathscr{A}(t) \\
& =z_{0}+Y(t)+\int_{E_{\cap}(0, t)} d A_{+}(s) .
\end{aligned}
$$


But $d A_{+}(s)=d Y_{-}(s)$, and $E=\left\{t ; \mathscr{A}(t)=-Y(t)-z_{0}\right\}=\{t ; Z(t)=0\}=$ $\{t ; \hat{\zeta}(t)=1\}$ so that

$$
Z(t)=z_{0}+Y(t)+\int_{0}^{t} \hat{\zeta}(s) d Y_{-}(s)
$$

showing that $Z$ is a solution of $\left(6^{\prime}\right)$.

Now let $Z$ be any other non-negative measurable solution of $\left(6^{\prime}\right)$. Then

$$
Z(t) \geqq z_{0}+Y(t) \text {. }
$$

Moreover, for any $s \leqq t$,

$$
Z(t)-Z(s)=Y(t)-Y(s)+\int_{s}^{t} \zeta(u) d Y_{-}(u) \geqq Y(t)-Y(s),
$$

and hence

$$
Z(t) \geqq Y(t)-Y(s) .
$$

It follows that $Z(t) \geqq Z(t)$. Thus $\zeta(t) \leqq \hat{\zeta}(t)$, and so

$$
Z(t)=z_{0}+Y(t)+\int_{0}^{t} \zeta(s) d Y_{-}(s) \leqq z_{0}+Y(t)+\int_{0}^{t} \hat{\zeta}(s) d Y_{-}(s)=Z(t),
$$

showing that $Z(t)=Z(t)$ and completing the proof.

We shall therefore take equation (6), with its unique solution (10), as the complete specification of the storage problem for an infinite dam with constant release. The solution (10) has been used by Gani and Pyke [3], who derived it by analogy with simpler models. Thus Gani and Pyke were, in effect, solving the problem with the release rule formulated here.

A consequence of equation (9) and of the proof of Theorem 1 is that $Z(t)$ satisfies

$$
Z(t)=z_{0}+Y(t)-\int_{0}^{t} \zeta(s) d Y(s)
$$

This equation does not, however, have a unique solution, and therefore cannot be used to specify the problem.

Equation (6) is equivalent to the original Gani-Prabhu equation (3) if and only if $Y_{-}(t)=\alpha t$. By virtue of (2), this occurs if and only if the Stieltjes measure determined by $X$ is singular with respect to Lebesgue measure, i.e. if and only if

$$
X^{\prime}(t)=0 \text { for almost all } t .
$$

This will occur, for instance, if $X$ increases only in jumps.

In connection with a related problem in the theory of queues, Beneš [1] has given a simple but useful identity, which can be generalised to the situation considered here. This we do in the following theorem.

THEOREM 2. Under the conditions of Theorem 1, and for any $\theta$, we have

$$
e^{-\theta Z\langle(t)}=e^{-\theta z_{0}-\theta Y(t)}-\theta \int_{0}^{t} e^{-\theta(Y(t)-Y(s)\}} \zeta(s) d Y_{-}(s) .
$$


Proof. Write

$$
C(t)=\int_{0}^{t} \zeta(s) d Y_{-}(s),
$$

so that $C$ is continuous, and

$$
Z(t)=z_{0}+Y(t)+C(t) .
$$

Then

$$
e^{-\theta C(t)}=1-\theta \int_{0}^{t} e^{-\theta C(s)} d C(s)=1-\theta \int_{0}^{t} e^{-\theta C(s)} \zeta(s) d Y_{-}(s) .
$$

But, when $\zeta(s) \neq 0$, we have $Z(s)=0$, and so $C(s)=-z_{0}-Y(s)$. Therefore

$$
e^{-\theta C(t)}=1-\theta \int_{0}^{t} e^{\theta z_{0} \div \theta Y(s)} \zeta(s) d Y_{-}(s),
$$

and so

$$
e^{-\theta Z(t)}=e^{-\theta z_{0}-\theta Y(t)-\theta C(t)}=e^{-\theta z_{0}-\theta Y(t)}-\theta \int_{0}^{t} e^{-\theta\{Y(t)-Y(s)\}} \zeta(s) d Y_{-}(s) .
$$

Hence the theorem is proved.

It now remains only to show how the analysis of the preceding section can be applied to the situation in which $X(t)$ is regarded as a stochastic process. We restrict attention to the case considered in [2] and [3], where $X(t)$ is a process with stationary independent increments having no deterministic component, and we write

$$
\boldsymbol{E}\left\{e^{-\theta X(t)}\right\}=e^{-t \xi(\theta)},
$$

where, by the Lévy-Khinchin representation theorem, $\xi(\theta)$ has the form

$$
\xi(\theta)=\int_{0}^{\infty}\left(1-e^{-\theta x}\right) d L(x),
$$

$L$ being a non-decreasing function.

It is a consequence of the Lévy-Itō theorem ([4], p. 553), that, with probability one, $X(t)$ increases only in jumps, of which there are finitely many in every finite interval if and only if

$$
\int_{0}^{\infty} d L(x)<\infty
$$

If the last condition is not satisfied, and in particular if the input is of Moran's gamma type [5], then the realisations of the process $X(t)$ are nondecreasing functions of quite a complex type. This is one of the reasons why Theorem 1 was proved for completely general inputs.

In any case, since $X(t)$ increases only in jumps, it must satisfy (12), so that $Y_{-}(t)=\alpha t$ and the identity (13) becomes

$$
e^{-\theta Z(t)}=e^{-\theta z_{0}} e^{-\theta Y(t)}-\alpha \theta \int_{0}^{t} e^{-\theta\{Y(t)-Y(\theta)\}} \zeta(s) d s .
$$


The process $Y(t)$ has stationary independent increments, and satisfies

$$
\boldsymbol{E}\left\{e^{-\theta \mathrm{Y}(t)}\right\}=e^{-t \eta(\theta)},
$$

where

$$
\eta(\theta)=\xi(\theta)-\alpha \theta \text {. }
$$

Now $\zeta(s)$ is determined by $Y(u)(u \leqq s)$, and is hence independent of $Y(t)-$ $Y(s)$. Thus, if $\theta \geqq 0$, we may take expectations in (18) to give

$$
\boldsymbol{E}\left\{e^{-\theta Z(t)}\right\}=e^{-\theta z_{0}} \boldsymbol{E}\left\{e^{-\theta Y(t)}\right\}-\alpha \theta \int_{0}^{t} \boldsymbol{E}\left\{e^{-\theta(Y(t)-Y(s)\}}\right\} \boldsymbol{E}\{\zeta(s)\} d s,
$$

or equivalently

$$
\boldsymbol{E}\left\{e^{-\theta \boldsymbol{Z}(t)}\right\}=e^{-\theta \boldsymbol{z}_{0}-t \eta(\theta)}-\alpha \theta \int_{0}^{t} e^{-(t-s) \eta(\theta)} \boldsymbol{P}\{Z(s)=0\} d s .
$$

This equation is exactly equivalent to equation (4.6) of [2], and we can now proceed as in that paper to determine the distribution of $Z(t)$. The resulting argument avoids any appeal to discrete time analogues, treating the problem throughout as one in continuous time.

I am greatly indebted to Mr. N. U. Prabhu and Dr. A. P. Robertson for stimulating discussions on the subject of this paper.

\section{References}

[1] Beneš, V. E., Combinatory methods and stochastic Kolmogorov equations in the theory of queues with one server. Trans. Amer. Math. Soc. 94 (1960) 282-294.

[2] Gani, J. and Prabhu, N. U., A storage model with continuous infinitely divisible inputs. Proc. Camb. Phil. Soc. 59 (1963) 417-429.

[3] Gani, J. and Pyke, R., The content of a dam as the supremum of an infinitely divisible process. J. Math. Mech. 9 (1960) 639-652.

[4] Loève, M., Probability Theory, (van Nostrand, 1960).

[5] Moran, P. A. P., A probability theory of a dam with a continuous release. Quart. J. Math. (Oxford) (2) 7 (1956) 130-137.

[6] Reich, E., Some combinatorial theorems for continuous time parameter processes. Math. Scand., 9 (1961) 243-257.

Universities of Cambridge and Western Australia. 